\title{
Investigation of non-inertial cavitation produced by an ultrasonic Horn
}

Peter R. Birkin, Douglas G. Offin, Christopher J. B. Vian

School of Chemistry, University of Southampton, Southampton UK SO17 1BJ. Email: prb2@soton.ac.uk

\section{Please cite this paper as:}

J. Acoust. Soc. Am, 2011 (130) , 3297-3308

The publisher's version of this paper is available here:

http://dx.doi.org/ 10.1121/1.3650537

\section{Related articles by Dr Peter Birkin can be found below:}

D. G.Offin, P. R. Birkin, T. G. Leighton, (2007). Electrodeposition of copper in the presence of an acoustically excited gas bubble. Electrochemistry Communications, 9, (5), 10621068. (doi:10.1016/j.elecom.2006.12.025).

A. O. Maksimov, T. G. Leighton, P. R. Birkin, (2008) Self focusing of acoustically excited Faraday ripples on a bubble wall. Physics Letters A, 372, (18), 3210-3216. (doi:10.1016/j.physleta.2008.01.041).

A. O. Maksimov, K. Winkels, P. R. Birkin, T. G. Leighton, (2008). Hopf bifurcation in acoustically excited Faraday ripples on a bubble wall. In Nonlinear Acoustics Fundamentals and Applications, Enflo, B. O.; Hedberg, C. M.; Kari, L., Eds. Vol. 1022, pp 229-232. (doi:10.1063/1.2956193).

A.Maksimov, T. Leighton, P. Birkin (2012) Acoustic microstreaming induced by pattern of Faraday waves on a bubble wall. The Journal of the Acoustical Society of America, 131, (4), 3338-3338. (doi.org/10.1121/1.4708495) 


\title{
Investigation of non-inertial cavitation produced by an ultrasonic
}

\section{horn}

\author{
Peter R. Birkin ${ }^{a *}$, Douglas G. Offin ${ }^{a}$, Christopher J. B. Vian ${ }^{a}$ \\ ${ }^{a}$ School of Chemistry, University of Southampton, Southampton, SO17 1BJ, UK. \\ E-mail:prb2@soton.ac.uk \\ Timothy G. Leighton ${ }^{b}$ \\ ${ }^{b}$ Institute of Sound and Vibration Research, University of Southampton, Southampton, \\ SO17 1BJ, UK. \\ Alexey O. Maksimov ${ }^{c}$ \\ ${ }^{C}$ Pacific Oceanological Institute, Far Eastern Branch of the Russian Academy of Sciences, \\ 690041 Vladivostok, Russia
}

Running title: Non-inertial cavitation by an ultrasonic horn

PACS Numbers: 43.35.Ei, 43.35.Ty, 43.25.Yw, 43.25.Ts, 43.35.Vz

Keywords: bubble, surface wave, mass transfer, ring-down

* author for correspondence 


\begin{abstract}
This paper reports on non-inertial cavitation which occurs beyond the zone close to the horn tip to which the inertial cavitation is confined. The non-inertial cavitation is characterised by collating the data from a range of measurements of bubbles trapped on a solid surface in this noninertial zone. Specifically, the electrochemical measurement of mass transfer to an electrode is compared with high-speed video of the bubble oscillation. This gas bubble is shown to be a "noninertial' event by electrochemical surface erosion measurements and 'ring-down' experiments showing the activity and motion of the bubble as the sound excitation was terminated. These measurements enable characterisation of the complex environment produced below an operating ultrasonic horn outside of the region where inertial collapse can be detected. Discussion is made of the extent to which solid boundaries in the liquid cause the frequencies and shapes of oscillatory modes on the bubble wall to differ from their free field values.
\end{abstract}




\section{Introduction}

The generation of sound has been used to influence chemical reactions and produce novel materials for many years ${ }^{1-3}$. In general, most of the investigations into this subject have concentrated on the generation of inertial ${ }^{4-6}$ cavitation (sometimes referred to as transient cavitation) through the employment of power ultrasound. In order to generate these inertial events (associated with sonoluminescence ${ }^{7,8}$, erosion $^{9-11}$, sonochemistry ${ }^{2,12,13}$ etc.) the conditions within the media must be suitable. In particular for water under normal conditions of temperature and pressure, the minimum conditions are that the Blake threshold ${ }^{6}$ and inertial limit (defined by Flynn ${ }^{4,5}$ and Apfel et al. ${ }^{14}$ ) must be exceeded in order to cause inertial cavitation. This entails a minimum zero-to-peak sound pressure amplitude of around $\sim 120 \mathrm{kPa}$ at $23 \mathrm{kHz}$ ultrasonic excitation to be present in water under normal conditions ${ }^{15}$. While this is achievable in many practical systems, regions of the liquid without this pressure amplitude will not possess these inertial events. However, it should be noted that inertial cavitation, although rich in its characteristics, is not the only bubble event that can be generated within these systems. Noninertial events (bubble motion, streaming etc.) are still likely to be present and excited by the acoustic environment. This implies that in order to understand the complete range of observed chemical and physical effects of cavitation, it is necessary to monitor the behaviour of gas bubbles including inertial and non-inertial events over a wide range of pressure conditions. The effect of non-inertial cavitation on fluid flow is the subject of this manuscript. In particular the behaviour of bubbles driven into oscillation by a $23 \mathrm{kHz}$ ultrasound source (an ultrasonic horn) but within a zero-to-peak pressure environment of $<120 \mathrm{kPa}$ is investigated using a combination of electrochemical, acoustic and high-speed imaging techniques. Bubbles driven into non-inertial cavitation by acoustic waves of the correct frequency and pressure amplitude, can undergo a number of different oscillations. These oscillations fall broadly into two classes: a breathing 
mode (or 'pulsation') where the whole bubble expands and contracts with spherical symmetry about the bubble centre; and a second class which lack spherical symmetry ${ }^{16,17}$.

The shape oscillations called Faraday waves are members of this second class ${ }^{18-20}$. A basic-level description is as follows. In the steady state, the breathing mode occurs at the frequency of the driving sound field, and this zeroth order spherical harmonic perturbation is the one which changes bubble volume, and hence the internal gas pressure, and so (through the pressure boundary condition at the bubble wall) dominates the far field acoustic radiation through a monopole emission. However, it is precisely because the zeroth order perturbation changes bubble volume (and so invests energy in pressure changes in the gas) that the associated wall displacements are small compared to the displacements that can be associated with shape oscillations, which do not change bubble volume, and hence do not generate significant far field acoustic radiation. However, as a consequence of the large wall displacement, the shape oscillations are amenable to visual and photographic observation ${ }^{21}$ and ultrasonic scattering ${ }^{22,23}$ and, furthermore generate significant liquid displacement, leading to liquid mixing ${ }^{24}$, microstreaming ${ }^{19,25,26}$ bubble 'dancing, ${ }^{27-29}$ and the 'shimmer' on the bubble wall ${ }^{30}$ which are often used as a visual indicator of achievement of the pulsation resonance ${ }^{31}$ (since maximizing the zeroth order wall displacements at resonance maximizes the chance of inducing the shape modes responsible for 'shimmering' and 'dancing').

Not unsurprisingly, the prediction of the natural frequency $f_{l}$ of an $l>1$ spherical harmonic (whether zonal or not) $Y_{l m}$ of order $l$ :

$$
f_{l}=\frac{1}{2 \pi} \sqrt{\frac{(l-1)(l+1)(l+2) \sigma}{\rho_{0} R_{0}^{3}}} \quad(l>1)
$$


is qualitatively different from (and generally lower than) the form predicting the zeroth order pulsation resonance frequency $f_{0}$ :

$$
f_{0}=\frac{1}{2 \pi R_{0} \sqrt{\rho_{0}}} \sqrt{3 \kappa\left(P_{0}+\frac{2 \sigma}{R_{0}}-P_{\mathrm{v}}\right)-\frac{2 \sigma}{R_{0}}+P_{\mathrm{v}}}
$$

where $\sigma$ is the surface tension, $\rho_{0}$ is the liquid density, $R_{0}$ is the spherically equivalent equilibrium bubble radius, $\kappa$ the polytropic index of the gas, $P_{0}$ is the static pressure in the liquid that would be present at the location occupied by the centre of the bubble if the bubble were not present, $P_{\mathrm{v}}$ is the vapour pressure of the bubble gas ${ }^{32}$. The zeroth order resonance of (2) can in fact be defined in slightly different ways ${ }^{33,34}$ depending on the parameter which is taken to peak (or locally peak) at resonance, for example scattered pressure, bubble wall velocity, or zero-topeak bubble wall displacement amplitude $R_{\varepsilon 0}$, which in the simple linear model of a spherical bubble is related to the zero-to-peak acoustic pressure of the incident wave $\left(P_{\mathrm{A}}\right)$ by $^{35}$ :

$$
R_{\varepsilon 0} \approx \frac{P_{\mathrm{A}} /\left(\rho R_{0}\right)}{\sqrt{\left(\omega_{0}^{2}-\omega^{2}\right)^{2}+\left(2 \beta_{t o t} \omega\right)^{2}}}
$$

where $\beta_{\text {tot }}$ is a damping factor with dimensions of time ${ }^{-1}$ which encompasses losses resulting from purely spherically symmetric motions ${ }^{34}$. Whilst this breathing mode is always excited when a bubble is insonified, the generation of each shape mode requires that this amplitude exceeds a threshold $^{30,36,37}$ given by $R_{\varepsilon 0, t}$ :

$$
R_{\varepsilon 0, t}=R_{0} \sqrt{\frac{(s-1)^{2}+4 p}{[-3 / 2 s+2 p+2(l+1 / 2)]^{2}+q^{2}}}
$$

where the terms $s, p$ and $q$ are given by: 
Birkin et al.

$$
\begin{gathered}
6=\frac{4(l-1)(l+1)(l+2) \sigma}{\rho \omega^{2} R_{0}^{3}} \\
p=\left[\frac{2(l+2)(2 l+1) \eta}{\rho \omega R_{0}^{2}}\right]^{2} \\
q=\frac{6(l+2) \eta}{\rho \omega R_{0}^{2}}
\end{gathered}
$$

Where $\eta$ is the shear viscosity. Since the amplitude, $R_{\varepsilon 0}$, is in most experiments not under direct control but rather is the response to other parameter settings (usually $P_{\mathrm{A}}$ and $\omega$ ), this threshold is more usually expressed as a threshold driving pressure such that the mode is excited when $P_{\mathrm{A}}$ exceeds the value $P_{l, \text { threshold }}$ that it takes when (3) achieves the amplitude given by (4).

The existence of a threshold for all modes $l>1$ means that at very low amplitudes only the breathing mode is excited, and at very high amplitudes many modes are excited. Within a narrow amplitude and frequency range, only the shape mode with the lowest threshold is excited, which is generally the one with a natural frequency closest to $f_{0} / 2$, and this is termed the 'Faraday wave'.

This simple model follows from analysis of the stability of the spherical gas bubble $27,38,39$ and has been subsequently tested ${ }^{37,40}$ (leading to the associated issue of mapping the parameter space for single bubble sonoluminescence ${ }^{41}$ ). There are complications to this simple picture, many associated with the interactions and coupling between modes ${ }^{37,42}$, the departure from low amplitude perturbations which can lead, for example, to bubble fragmentation ${ }^{43}$, occasions when other subharmonics and ultraharmoncs characterise the shape mode with the lowest threshold ${ }^{27}$, the influence of the contact line when the bubbles exhibiting these modes touch other surfaces (as here ${ }^{44,45}$, hysteresis ${ }^{29}$, and the role of viscosity. Viscosity is not present in (1) but clearly should influence the effects of microstreaming and the characteristic shape of the threshold curve, producing very different effects from those associated with the breathing mode ${ }^{46,47}$. Despite 
these complications, the simple scheme presented in (1)-(4) is sufficient to explore the experimental observations made in this paper.

The data presented in this paper is concerned with electrochemical and photographic detection of bubble oscillation and Fig. 1 shows a collection of results detailing previous electrochemical ${ }^{20,30}$ and photographic observations. Note that in Fig. 1 a relatively large gas bubble has been held by buoyancy forces beneath a solid surface. This bubble is then driven into oscillation by irradiation with sound. The distortions in the surface of the bubble can be clearly seen. The contribution to mass transfer of material from the bulk liquid to a solid/liquid interface can be quantified using a microelectrode positioned close to the gas/liquid interface of the bubble. Microelectrodes were chosen for these studies for a number of reasons. These included their ability to operate under steady state conditions (e.g. where, under stagnant conditions, the concentration profile is time invariant), their relatively fast response time and their small size. In these experiments an $\mathrm{XYZ}$ micrometer and stage were used to position a microelectrode close to the gas/liquid interface of an air bubble trapped by buoyancy forces under a solid surface. The position of the microelectrode with respect to the gas/liquid interface was verified by monitoring the hindered steady state current (the current detected under mass transfer control) recorded in the absence of bubble oscillation $^{30}$. This reduction in current was due to negative feedback ${ }^{48}$ as a result of the blocking nature of the gas/liquid interface under the conditions stated. Irradiation of the bubble with sound of the appropriate frequency and amplitude results in oscillation of the bubble surface. This oscillation can be electrochemically detected by the microelectrode as an enhancement in the mass transfer limited current as a result of the forced convection (or microstreaming) of the liquid produced by the oscillation of the bubble wall. Fig. 1c shows this enhancement in mass transfer to the microelectrode as a function of distance away from the gas/liquid interface of the bubble over an extended range ${ }^{20}$ (up to $2500 \mu \mathrm{m}$ ). In this case, the bubble was driven into 
oscillation at a pressure sufficient to generate surface waves on the gas/liquid interface. This was observed in two ways. First, the presence of surface waves on the bubble wall was observed optically as a 'shimmer'. Second, when the microelectrode was positioned close to the bubble wall $(c a .5-10 \mu \mathrm{m})$ the motion of the bubble wall can be resolved electrochemically. This is shown as an insert on Fig. 1c. The insert shows the current and pressure time traces for a bubble driven to oscillate with surface motion (note that the absolute pressure amplitude of the driving field can only be measured after the bubble has been removed from the liquid). This clearly shows that the current time trace has a component at $0.5 f$ (where $f$ represents the drive frequency, in this case $1.46 \mathrm{kHz}$ ). This is characteristic of Faraday waves on the surface of the bubble wall, and is confirmed by the photograph of a bubble under similar conditions shown in Fig. 1. Fig. 1 also shows that within the first $c a .100 \mu \mathrm{m}$, the mass transfer enhancement remains approximately constant, whilst at greater separation the current falls as the distance between the microelectrode and the gas/liquid interface of the bubble increases. These investigations have shown that combining electrochemical measurements with imaging and acoustic characterisation of the system can be extremely powerful for the elucidation of mechanistic detail associated with bubble oscillation. However, these investigations can also be extended to the study of general bubble behaviour in a cavitation plume produced by an operating ultrasonic horn. It should be noted that acoustic characterisation and experimental investigation suggests that, outside of the 'inertial zone', there is still significant pressure amplitude (of the order of $30 \mathrm{kPa}$ at $4.5 \mathrm{~mm}$ separation from the sound source) within the liquid. This observation suggests that non-inertial motion of gas bubbles should be observable. Here we show that this is possible and results in the trapping of oscillating gas bubbles at the solid/liquid interface.

\section{Experimental}


Ultrasound and cavitation were generated by means of a Grundig Digimess FG 100 function generator, Brüel \& Kjær Type 2713 power amplifier and ultrasonic transducer fitted with a $3 \mathrm{~mm}$ diameter titanium tip (Adaptive Biosystems) ${ }^{9,15,49}$. The function generator was interfaced with a PC using software written in-house, allowing the frequency, power and duration of the ultrasound to be accurately controlled. The acoustic measurements were the pressure time histories (measured by a Brüel \& Kjær Type 8103 calibrated hydrophone). The exact conditions for each experiment are given in the figure captions. Although calorimetry was not used as a primary measure (as it is only an indirect proxy $^{50}$ for source power), the ratio of that nominal power to the faceplate area $\left(0.071 \mathrm{~cm}^{2}\right)$ as determined in the usual manner ${ }^{51}$ was $\sim 50 \mathrm{~W} \mathrm{~cm}^{-2}$.

For electrochemical measurements, the working electrode was inserted in the bottom of an electrochemical cell, which itself was placed on an XY stage (Photon Control). The stage allowed $25 \mathrm{~mm}$ of movement in each direction with $0.01 \mathrm{~mm}$ resolution, enabling the position of the working electrode to be accurately controlled in a plane below the tip of the ultrasonic horn. The position of the horn was fixed in the XY plane but controlled in the $\mathrm{Z}$ direction by means of a micrometer and stage (Newport), which allowed $25 \mathrm{~mm}$ of movement with $0.02 \mathrm{~mm}$ resolution. This allowed the separation between the surface of the working electrode and the tip of the ultrasonic horn to be controlled with a high degree of precision. This is a key experimental requirement for obtaining reproducible results from acoustoelectrochemical studies. Figure 2 shows a schematic representation of the experimental set-up employed here. In this example a bubble, driven by an appropriate acoustic field, is trapped on the surface of the electrode support (by radiation forces for example) and enhances local forced convection which can be detected by the microelectrode as an increase in electrochemical current (with periodicity related to the fluid motion generated for example) as a function of time. The nature of the electrode materials, the 
electrode potential (controlled with respect to a reference electrode) and the solution makeup will dictate the response of the electrode. In turn the physical effects investigated can be varied between surface erosion (for example using a passive lead electrode) and mass transfer (for example convection and diffusion) to a platinum electrode. Hence, in order to gain further information on the local processes driven by the bubbles/sound-field, it is convenient to employ a 'dual electrode'. In this case the electrode support contains two electrodes with differing character; a lead electrode for surface erosion measurement in sulphate media and a platinum electrode for measurement of mass transfer effects. The area over these electrodes can also be imaged using a high-speed camera in order to gain further insight on the mechanisms generated.

Current from the dual electrodes was measured using a two channel current follower constructed in house. High-speed video footage was recorded using a Photosonics Phantom V7 digital video camera fitted with a monozoom lens. The camera is capable of recording at up to 150000 frames per second depending on the resolution selected by the user. Chemicals, such as $\mathrm{K}_{4}\left[\mathrm{Fe}(\mathrm{CN})_{6}\right]$ (Aldrich, 99\%), $\mathrm{Na}_{2} \mathrm{SO}_{4}(\mathrm{BDH}$, AnalaR), $\mathrm{KI}$ (Timstar, Analytical) and $\mathrm{KCl}$ (Fischer, Analytical), were used as received. Purified water was obtained from a USF Elga Purelab Option 10 system (water obtained from this system had a total organic carbon, according to the manufacturer, of $<30 \mathrm{ppb}$ and a resistivity $>15 \mathrm{M} \Omega \mathrm{cm}$ ).

\section{Results}

Evidence for inertial collapse has been shown and described within the first few $(<2.5 \mathrm{~mm})$ millimetres from the $3 \mathrm{~mm}$ diameter ultrasonic horn employed ${ }^{15}$. Clearly, although this experimental evidence and the associated mechanisms are extremely interesting, they only occupy a relatively small volume of the liquid (here up to only $0.02 \mathrm{~cm}^{3}$ ). Under the conditions 
employed here, at distances greater than $\sim 2.5 \mathrm{~mm}$ from the tip of the ultrasonic horn, erosion of electrode surfaces (which is associated with inertial cavitation) has been shown to terminate ${ }^{11,15}$. However, significant enhancements in the rate of mass transfer to an electrode can be measured up to $2 \mathrm{~cm}$ from the operating ultrasonic horn. At these greater distances, earlier authors detected regular spikes in the electrochemical current and suggested, through the use of numerical simulations, a violent bubble collapse or oscillation at harmonics of the driving frequency ${ }^{52,53}$ near the electrode surface (e.g. 10's nm with bubble wall velocities in the region of 160-320 m s' $\left.{ }^{1}\right)$. In addition by considering the way the thin layer of viscous liquid between the bubble and the electrode must evolve during cavitation, they inferred that local pressure amplitudes of 30-250 $\operatorname{bar}^{52}$ would be required by considering the timescales for evolution of the bubble wall and diffusion layer at the electrode. While these estimations are of interest, these high pressures (given the distance from the horn surface and quoted intensities) and wall velocities suggest this system requires further investigation. This is done here in a set of experiments where electrochemical data is combined with high-speed imaging. In addition a 'ring-down' situation, where current transients are detected after the horn has been turned off is demonstrated. As a result of the collection of experiments, current transients at extended distances from the ultrasonic source (shown to be outside the inertial zone for the sound source employed) are attributed here to non-inertial oscillations of a stable semi-permanent gas bubble moving over the surface of the electrode.

Figure 3(a) shows a typical current time trace recorded under conditions where inertial cavitation is not present. Here a dual electrode was employed ${ }^{10,54}$. This consisted of a $\mathrm{Pb}(125 \mu \mathrm{m}$ diameter) and $\mathrm{Pt}$ (50 $\mu \mathrm{m}$ diameter) electrode sealed in close proximity to one another in epoxy resin (centre-to-centre distance $\sim 100 \mu \mathrm{m}$ ). This electrode, through careful choice of the solution constituents, electrode material and the electrode potential, is able to detect surface erosion on the 
$\mathrm{Pb}$ (associated with inertial cavitation) and mass transfer enhancements (associated with streaming, microstreaming and other bubble induced forced convection phenomena) at the Pt electrode surface ${ }^{10}$. A key feature here is that Fig. 3 shows that although a repetitive signal is seen on the Pt electrode, no signal is observed on the $\mathrm{Pb}$ electrode. This suggests that the mechanism responsible for the mass transfer enhancement is reliant on non-inertial cavitation as it generates no erosion ${ }^{4-6}$. In order to investigate the origin of this periodic signal, a high-speed camera was used to probe the environment above the electrode under these conditions. Fig. 3(b) shows a sequence of images recorded under such conditions. These images show a bubble moving around on the surface and pulsating periodically. However, as will be shown here, this is not a transient/inertial cavitation event (as indicated by the absence of an electrochemical erosion signal) but a gas bubble trapped on the surface of the electrode. In order to understand the behaviour of the gas bubble, a 'ring-down' experiment was performed in conjunction with highspeed imaging of the system.

In the ring-down experiment, the ultrasound was turned off when an event such as that shown in Fig. 3(a) was detected. The acoustic pressure and current were then measured as a function of time and high-speed video was also recorded simultaneously. This is particularly useful technique for examining the individual modes when many are excited simultaneously, since the damping of each mode differs ${ }^{55}$, and therefore so do their decay times. Figure 4 shows the evolution in behaviour of the signal obtained from a hydrophone placed in the cell in conjunction with measurement of the electrochemical signal from a $25 \mu \mathrm{m}$ diameter Pt electrode. The cessation of sonication (here at $15 \mathrm{~ms}$ ) leads to a gradual decrease in the acoustic pressure amplitude as the probe and cell 'ring-down' (e.g. the acoustic pressure within the cell falls as a result of acoustic absorption). The pressure field within the cell appears to reach a background level after $\sim 100 \mathrm{~ms}$. The current is relatively high initially (at $t=0$ ), which is to be expected as 
the data acquisition was triggered by the detection of such events. However, even after the ultrasound is terminated the electrode detects significant changes in local mass transfer (these are labelled A-D on Fig. 3b). This indicates that during this $85 \mathrm{~ms}$ period, fluid motion around the electrode is significant but transient in nature.

Further information is gathered from the high-speed imaging recorded simultaneously with the pressure and current time history shown in Fig. 4(a), (b). In this case the electrode/solution interface was imaged at 100000 frames per second (fps). Figure 5 shows every $50^{\text {th }}$ frame i.e. the 200 frames corresponding to the data shown in Fig. 4 (note the frame number is included in Fig. 4(b) for reference). It is important to note that the actual electrode is the dark line left of centre at the bottom of each frame, which is encased in a transparent support on which the bubble is sitting. The boundary between this support and the solution is slightly blurred.

In the first $15 \mathrm{~ms}$ (frames 1 to 30 ) the ultrasound is on and a large bubble or cluster of bubbles can be seen at the surface. The bubble (or cluster of bubbles) is oscillating in size (compare for example frames 6 and 7) and also there is translational motion across the surface. During this time the current recorded at the electrode is relatively high. The current remains high for $2 \mathrm{~ms}$ (until frame 32) and then starts to fall. The current reaches a local minimum and stays low until frame 47, when it rises again to a peak (labelled A) at frame $50(t=25 \mathrm{~ms})$. It can be seen in Fig. 5 that the drop in current after frame 32 is associated with the translation of the bubble away from the electrode and out of frame to the left-hand side. During the period of low current there is no bubble visible in the frame. The bubble re-enters the left hand side of the image in frame 47 and is directly on top of the electrode in frame 50, corresponding to peak $A$ in Fig 4(b). Following peak $A$ there is a decrease in the current followed by a rise to peak $B$ at frame $58(t=29 \mathrm{~ms})$. The decrease after peak $A$ is associated with the bubble moving out of focus (see frames $51-54$ ) and then to the left (frames 55 and 56) indicating that it has moved away from the electrode 
(which is in the plane of focus). The bubble then comes back into the frame and focus (or better focus) in frames 57-58, which corresponds to peak B. Although the bubble being blurred could indicate rapid motion rather than the bubble moving out of the plane of focus, this is unlikely as the bubble is resolved more clearly between frames 1 and 30 , when the velocities would be expected to be higher, than frames 51 to 54 as the system is still under insonification up until frame 30. It is also important to note that while the bubble is out of focus, large shape oscillations can still be seen. This suggests that the bubble needs to be close ( $\sim 1$ bubble radii) to the electrode for the mass transfer enhancement to be detected, which is in agreement with previous work $^{20}$. Following peak B there is another decrease in the current until frame 80 , when the current starts to increase. Between frame 58 and 80 the bubble is either out of focus or not in the frame, indicating that it is not near the electrode and hence the does not enhance mass transfer to the surface significantly so no elevated current is seen. After the rise in current at frame 80 it stays relatively high until frame $90(t=40-45 \mathrm{~ms})$, peak $\mathrm{C}$. As with peaks $\mathrm{A}$ and $\mathrm{B}$, peak $\mathrm{C}$ (frames 81 to 90 ) is associated with a bubble appearing on or near the electrode and in focus. The bubble can be seen to change shape and size and the elevated current indicates that this leads to enhanced mass transfer of material to the electrode surface. Following the period of elevated current there is another decrease until a minimum at frame $110(t=55 \mathrm{~ms})$. Again this decrease corresponds to frames in Fig. 5 where the bubble appears out of focus (frames $90-110$ ) and hence some distance from the electrode. The current then rises slowly (starting around frame 113), through a broad peak (labelled D) and decays back to a background level (frame 165). Throughout the frames that encompass peak D (113 to 165) the bubble can be seen to move into focus and then out of focus again, indicating that the bubble translates across the electrode surface. Also throughout there are shape oscillations present but as in the above discussion the current is only enhanced when the bubble is close to the electrode. 
It is interesting to compare peaks $A$ and $D$ in terms of the evolution of bubble behaviour during the experiment. Peak $A$ is sharp and high whereas peak $D$ is broad and low. The translational velocities associated with peak $A$ are high compared with peak $D$. The bubble moves from out of the picture in frame 47 to the centre of the picture in frame 50. The bubble is in focus in frame 50 and out of focus in frame 51 indicating that the bubble has again moved rapidly. In contrast for peak $D$ the bubble moves in and out of focus over 10's of frames indicating that the translational velocity is much less. Clearly the environment at the start of the ring-down experiment is very dynamic and becomes less so as the experiment progresses. This was investigated further through high-speed imaging.

Figure 6 shows a set of images recorded at different times through a ring-down experiment. The frame rate was $100000 \mathrm{fps}$ and every frame is shown so the interframe time is $10 \mu \mathrm{s}$ compared with $500 \mu$ s for Fig. 5. Again a large bubble can be seen on the surface of the electrode which undergoes large amplitude pulsation and motion across the solid/liquid interface of the electrode housing. Figure 6a was recorded when the sound field was still active. Note the ring-down experiments were performed so that $15 \mathrm{~ms}$ of data was recorded while the ultrasonic horn is actively driven. Figure 6a shows a cloud or cluster of bubbles moving across the surface of the glass/liquid interface of the microelectrode housing. The shape of the bubble cluster is apparently compressed towards the surface of the electrode and clear fragmentation can be seen (see Fig. 6a frame 65-67). Figure $6 \mathrm{~b}$ shows the bubble event $\sim 12 \mathrm{~ms}$ after termination of the drive signal to the ultrasonic transducer (i.e. conditions similar to those under which peak $A$ was observed). Again the bubble cluster can be seen to be dynamic in nature. However, as time progresses the motion of the bubble become less complex. For example Fig. 6(c) shows a single bubble with surface distortions on the gas/liquid interface. In this set of images the drive to the transducer was terminated $\sim 52 \mathrm{~ms}$ before the images were captured. Figure 4(a) shows that after 
this order of time, ring-down of the sound field is significant. However, the motion of the gas bubble can be detected electrochemically (see Fig. 4b transient D). Finally, Fig. 6(d) shows a set of images recorded $\sim 67 \mathrm{~ms}$ after termination of the drive signal to the transducer. In this case, it appears as if a single surface wave is present on the gas/liquid interface, although three independent viewpoints would be needed to confirm this.

When the pressure drops below the threshold required to generate a single surface wave and all such wave motions have damped out (not shown), the bubble undergoes only spherical symmetric pulsations until no motion could be observed. The amplitude of these spherical pulsations was estimated to be on the $\mu \mathrm{m}$ scale through modelling of bubble behaviour ${ }^{56}$. Eventually (after $\sim 100 \mathrm{~ms}$ ) the acoustic pressure amplitude falls to essentially zero. However, a gas bubble remained on the surface of the electrode. In this case the bubble was estimated to be $220 \pm 20 \mu \mathrm{m}$ in diameter.

\section{Discussion}

\section{A Identification of mode}

The high-speed imaging shows a range of bubble behaviour. It is helpful to consider the latter stages of oscillation first, because at that time a clear identification of the bubble mode can be successfully made. The extreme of the oscillation in Fig. 6d (achieved at an interval of a little less than 9 frames) appears to take the form of a 5 pointed star (see frames $9,18,27,36,44,52$, $61,70,79,87)$. To show how well the shape appears to conform to an $l=5$ zonal spherical 
harmonic perturbation $Y_{5}^{0}$, assume that in the absence of the independent viewpoints we have a vertical axis of symmetry, and plot out a radius perimeter of

$R=R_{0}\left(1+a_{5,0} Y_{5,0}(\theta)\right)$

$Y_{5,0}(\theta)=\frac{1}{16} \sqrt{\frac{11}{\pi}}\left(63 \cos ^{5} \theta-70 \cos ^{3} \theta+15 \cos \theta\right)$

where $a_{l, m}$ is the amplitude weighting of the spherical harmonic perturbation $Y_{l, m}$. Here a value of $a_{5,0}=0.13$ produces the good fit to frame 79, as shown in Fig. 7(a). However, the 9 oscillations between frames 9 and 87 take 78 frames, that is, $780 \mu$ s, implying a period of $\sim 87 \pm$ $0.3 \mu$ s and hence a mode frequency of $f_{l} \sim 11.5 \pm 0.04 \mathrm{kHz}$.

This provides a useful example of the care which needs to be applied when assigning the mode number from the imaging data alone. Consider equations (1)-(6), and the parameters relevant to Fig. 6(d) (see Table 1). With these input values, equation (1) predicts $f_{4}=11.5 \pm 1.6 \mathrm{kHz}$, $f_{5}=15.7 \pm 2.1 \mathrm{kHz}$, and $f_{6}=20.3 \pm 2.8 \mathrm{kHz}$, indicating that the observed mode frequency agrees best with that calculated for mode number $l=4$. However, this would appear to contradict the pentagonal shape observed in Fig. 6(d) which would suggest $l=5$. In order to resolve this conflict, appreciation of the symmetry and pattern formation on the bubble wall is necessary. Note that a basic feature of pattern formation, which is applicable for the interpretation of preferred patterns of parametrically unstable Faraday ripples on the sphere, is that these structures have symmetry of point subgroups including the symmetries of Platonic solids ${ }^{47,57}$. The maximal symmetry group for $l=4$ is octahedral, and Fig. $7(\mathrm{~b})$ shows the pattern corresponding to this symmetry, the example showing the case where several $l=4$ modes are superimposed on the sphere, and the axial of symmetry no longer holds. The cube symmetry assumes the presence of three 4-fold axes, one of these being the vertical z-axis. Consequently only the $Y_{4,4}, Y_{4,-4}$ and $Y_{4,0}$ modes (i.e. $l=4$, and $m=-4,0,4$ ) can form the octahedral structure ${ }^{57}$. The first two modes form a standing wave and have equal amplitudes (i.e. $a_{4,4}=a_{4,-4}$ ). The equality 
$a_{4,0}=\sqrt{14 / 5} a_{4,4}$ follows from the requirement that rotation by $\pi / 2$ radians about the $x$ - and $y$ axes should leave the structure unchanged.

The periodicity therefore indicates $l=4$ through Eq. (1), whilst the mode shape suggests $l=5$. To resolve this apparent discrepancy, consider that the physics that is omitted in applying Eqs. (1) and (7) to the data includes the presence of the solid boundary. High-speed imaging of such small objects made it difficult to image the contact conditions, but if a contact angle of around 0.44 radians (Fig. 7(a)) is assumed, the extra inertia would decrease the frequency of the breathing mode by a factor of $\sim 0.8$ compared to its free field value $e^{45,58}$. Application of a similar scaling factor would produce modified frequencies $f_{n}^{\prime}$ of $f_{4}^{\prime}=9.4 \pm 1.3 \mathrm{kHz}, f_{5}^{\prime}=12.8 \pm 1.3 \mathrm{kHz}$, and $f_{6}^{\prime}=16.6 \pm 2.3 \mathrm{kHz}$. This would apparently reconcile the mode frequency with the apparent $l=$ 5 shape of Fig. 7(a), but there is as yet no justification for applying a correction factor of similar size as would pertain to the breathing mode. Indeed the expectation would be for a much smaller reduction in shape mode frequency than breathing mode frequency, since for volume oscillations the potential falls off as $r^{-1}$ (where $r$ represents the distance from the bubble centre) and the inertial mass is located in a layer of liquid of width $\sim R$ (where $R$ is the bubble radius) near the bubble wall. In contrast, the potential for the mode $l$ will fall off as $r^{-l+1}$ and the inertial mass of these modes will be localized in layers of liquid near the bubble wall that become ever thinner as the mode order increases. Moreover, this shift will be different for the modes with a fixed degree $l$ and different values of $m$. The mode $\{l l\}$, localized near the equator, will not experience the influence of the boundary located near the pole and vice versa. The mode $\{l 0\}$ will be most affected by the boundary at the poles. If, instead of being in an infinite body of liquid, the bubble becomes close to a rigid wall, then the effect of the wall would be equivalent to the presence of a mirror bubble which oscillates (in comparison to the source bubble) with the phase and symmetry shown in Fig. 7(c). Because of the $r^{-l+1}$ decay in potential away from the bubble wall and the 
restriction of the inertia to thin layers just outside of the bubble wall, the mirror bubble will perturb only a small region of liquid in the neighbourhood of the contact area. Consequently, as $l$ increases, the presence of the wall will decrease the natural frequencies of the mode to ever decreasing extents, compared to the free field value. Hence the correction factor for the $l=4$ or 5 modes of this bubble can be expected to be less than the $\sim 0.8$ which would apply to the breathing mode frequency. Hence, consideration of the argument described above would suggest that the presence of the wall would not reduce the $l=5$ mode frequency to the $11.5 \pm 0.04 \mathrm{kHz}$ observed experimentally, and that instead the observed periodicity agrees with the $l=4$ mode frequency. The discrepancy between the observed bubble shape (Fig. 7(a)) and the one calculated for the $l=$ 4 mode (Fig. 7b) is reconciled in the panel on the right of Fig. 7(c), which indicates how the presence of the mirror bubble that represents the wall can give the $l=4$ mode the appearance of a free field $l=5$ mode. The lobes of the bubble distortions directed to the wall will decrease as the bubble approaches the wall, since the mirror bubble will suppress these oscillations. The side lobes will be slightly deformed to move closer to the $z$-axis owing to the influence of the mirror bubble. Finally, when the bubble reaches the wall, silhouette of the $l=4$ mode when viewed from the side corresponds to a pentagon. This is a more likely explanation for the shape of the bubble in frame 79 of Fig 6(d) than is the existence of the $Y_{5,0}$ mode plotted in Fig. 7(a). Indirect evidence is also obtained from observation of the movie associated with the Fig. 6(d) stage of the ring-down ${ }^{59}$. In addition, other reports ${ }^{40,60}$ investigating acoustically excited bubbles within fluids have suggested octahedral shapes for parametric resonances of $l=4$ (see for example Fig. 2, 3 of Versluis et al. $)^{40}$. The final indirect evidence that the mode in Fig. 6(d) does not correspond to $l=5$ is that, if $Y_{5,0}$ were present, there would be a liquid layer between the bubble and the wall, and displacements ('dancing') of the bubble over the wall: during this stage of the 
Birkin et al.

ring-down the bubble does not dance ${ }^{59}$. Taken together, these three observations indicate that $l=$ 5 is not present in Fig. 6(d), and that instead the oscillations correspond to $l=4$.

\section{B Progression through modes during ring-down}

Having in the previous subsection seen the care which must be applied when interpretting these data of bubbles close to walls with the idealised free field theory of Eqs. (1)-(6), the ring-down behaviour will now be discussed in terms of that theory with due reservations about exact correspondences. In order to predict the behaviour of the gas bubble, it is necessary to quantify a number of physical parameters applicable to either the gas or liquid phase of the system. The values used are quoted in table 1. Hydrophone measurements and sound field modelling indicate that, during steady state inconification, the zero-to-peak acoustic pressure amplitude of the 22.85 $\mathrm{kHz}$ signal from the ultrasonic source is approximately $30 \mathrm{kPa}$ at the electrode surface, in agreement with earlier estimations. ${ }^{15}$ Figure 8 shows the estimated pressure thresholds for a number of surface wave modes on a $220 \mu \mathrm{m}$ diameter bubble under the conditions stated. Figure 8 point ' $A$ ' shows the initial pressure conditions expected from hydrophone measurements while the sound source is operational. Note that five of the six surface waves considered (here order $l=$ $2-7$ ) are active at point $A$ in Fig. 8. Under these conditions the behaviour of the bubble will be complex with multiple surface waves active at one time. This is likely to contribute to the complex bubble/cluster event (including fragmentation) seen in Fig. 6(a). However, as the ringdown experiment proceeds, the acoustic measurements (see Fig. 4(a)) indicate that the pressure amplitude at the surface of the electrode will fall. Under these circumstances the behaviour of the gas bubble progressively becomes less complex. There are two timescales; that for the sound field to decay, and that for each mode to decay. If these differed by orders of magnitude, the 
picture would be simple: if, for example, the sound field decayed rapidly compared to the modal decay times, the ring-down images would purely represent modal decay rates, but the pressure measurements indicate that this is not the case. For example, measurements of the pressure ringdown within the electrochemical cell (not shown) indicate a reverberation time (T60) of $83.2 \mathrm{~ms}$. Under these conditions the sound field takes around $44 \mathrm{~ms}$ to decay to $\sim 1 \mathrm{kPa}$. However, the high-speed imaging (see Fig. 6) suggests that the surface waves are still active and ring-down over a longer time period (here $92 \mathrm{~ms}$ for complete surface wave activity to cease). Hence it is likely that each mode decays at its characteristic rate once the local pressure in the vessel falls below its threshold for that mode and frequency. For example by the time the pressure amplitude has fallen to $15 \mathrm{kPa}$ (see point $\mathrm{B}$, Fig. 8) only three surface wave modes $(l=3,4,5)$ may be directly excited (although the higher modes may be ringing-down). The corresponding motion of the gas bubble is likely to be less complex compared to the initial situation in Fig. 8 point A. It is plausible that this corresponds to Fig. 6(c), where multiple surface waves are observable. As the pressure amplitude at the electrode falls further to say $5 \mathrm{kPa}$, all higher modes ring down to leave a single surface wave excited (see Fig. 8 point C) on the bubble wall. Finally this single mode predicted to be of order $l=4$ (see Fig. 7), as confirmed in the previous subsection, is observed on its own. When the pressure amplitude falls below $\sim 1 \mathrm{kPa}$ (see Fig. 8 point D), no surface waves can be excited, the last shape mode decays away and the bubble is left to oscillate spherically symmetrically until all motion ceases.

The pressure measurements, acoustic modelling and lack of an erosion signal from the electrochemical experiments indicates that the periodic signals observed here are likely to be due to this trapped bubble and not due to inertial cavitation.

Clearly the environment generated below an operating ultrasonic horn is both complex and fascinating. It should be noted that the presence of the electrode is far from non-invasive 
Birkin et al.

(see for example ref ${ }^{54}$ ) and will interact with the sound field generated by the cavitation process in particular. Although useful information can be gathered, this is a fundamental complication to electrochemical investigations of these systems.

\section{Conclusions}

An electrochemical approach in combination with high-speed imaging and acoustic measurements has been used to study the environment below an operating ultrasonic horn outside of the inertial cavitation zone. This environment has been shown to shown to produce bubbles held above an electrode support by streaming and radiation forces. These surface confined bubbles oscillate and move over the substrate to produce multiple electrochemical mass transfer events. Ring-down experiments have shown the presence of surface waves on the gas/liquid interface. After cessation of insonification, the sound field decays, as do the modes (at different characteristic rates). Care is required in the identification of the last shape mode since the shape itself can be misleading, and simultaneous visualisation from three orthogonal directions is recommended. The threshold for these events is of the order of $1 \mathrm{kPa}$ and occurs due to Faraday wave motion and higher modes of a permanent gas bubble associated with the surface.

\section{Acknowledgements}

We thank the EPSRC for funding (EP/E024408/1 and EP/D05849X/1) and the EPSRC Instrument Loan Pool for assistance with the high-speed imaging reported in this manuscript. 


\section{Birkin et al.}

\section{References}

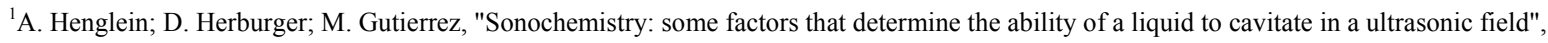
Journal of Physical Chemistry. 96, 1126-1130, (1992).

${ }^{2}$ K. S. Suslick, "Sonochemistry", Science. 247, 1439-1445, (1990).

${ }^{3}$ A. Weissler; H. W. Cooper; S. Snyder, "Chemical effect of ultrasonic waves: oxidation of potassium iodide solution by carbon tetrachloride", Journal of the American Chemical Society. 72, 1769-1775, (1950).

${ }^{4}$ H. G. Flynn, "Cavitation Dynamics II. Free pulsations and models for cavitation bubbles", Journal of the Acoustics Society of America. 58, 11601170, (1975)

${ }^{5}$ H. G. Flynn, "Cavitation Dynamics I. A mathematical formulation", Journal of the Acoustics Society of America. 57, 1379-1396, (1975).

${ }^{6}$ T. G. Leighton "The Acoustic Bubble, section 4.3.1"; (Academic Press, London, 1994); 312-329.

${ }^{7}$ A. J. Walton; G. T. Reynolds, "Sonoluminescence", Advances in Physics. 33, 595-660, (1984).

${ }^{8}$ D. F. Gaitan; L. A. Crum; C. C. Church; R. A. Roy, "Sonoluminescence and bubble dynamics for a single, stable, cavitation bubble", Journal of the Acoustical Society of America. 91, 3166-3183, (1992).

${ }^{9}$ P. R. Birkin; D. G. Offin; T. G. Leighton, "The study of surface processes under electrochemical control in the presence of inertial cavitation", Wear. 258, 623-628, (2005).

${ }^{10}$ P. R. Birkin; D. G. Offin; T. G. Leighton, "A novel dual microelectrode for investigating mass transfer and surface erosion caused by cavitation", Electrochemistry Communications. 6, 1174-1179, (2004).

${ }^{11}$ P. R. Birkin; R. O'Connor; C. Rapple; S. Silva-Martinez, "Electrochemical measurement of erosion from individual cavitation generated from continuous ultrasound", Journal of the Chemical Society Faraday Transactions. 94, 3365-3371, (1998).

${ }^{12}$ L. H. Thompson; K. Doraiswamy, "Sonochemistry: Science and Engineering", Industrial Engineering Chemical Research. 38, 1215-1249, (1999).

${ }^{13}$ C. von Sonntag; G. Mark; A. Tauber; H.-P. Schuchmann. Advances in Sonochemistry; JAI Press, 1999; Vol. Vol. 5; pp 109-145.

${ }^{14}$ C. K. Holland; R. E. Apfel, "An Improved theory For the Prediction of Microcavitation Thresholds", IEEE Transactions Ultrasonics Ferroelectrics and Frequency Control. 36, 204-208, (1989).

${ }^{15}$ P. R. Birkin; D. G. Offin; T. G. Leighton, "Experimental and theoretical characterisation of sonochemical cells. Part 2: cell disruptors (Ultrasonic horns) and cavity cluster collapse", Physical Chemistry Chemical Physics. 7, 530-537, (2005). 


\section{Birkin et al.}

${ }^{16}$ T. G. Leighton; A. D. Phelps; D. G. Ramble; D. A. Sharpe, "Comparison of the abilities of eight acoustic techniques to detect and size a single bubble", Ultrasonics. 34, 661-667, (1996).

${ }^{17}$ A. D. Phelps; D. G. Ramble; T. G. Leighton, "The use of a combination frequency technique to measure the surf zone bubble population", Journal of the Acoustical Society of America. 101, 1981-1989, (1997).

${ }^{18}$ M. Faraday, "On a peculiar class of Acoustical Figures; and on certain Forms assumed by groups of particles upon vibrating elastic surfaces", Philosophical Transactions of the Royal Society London. 121, 299-340, (1831).

${ }^{19}$ S. A. Elder, "Cavitation microstreaming", Journal of the Acoustical Society of America. 31, 54-64, (1959).

${ }^{20}$ P. R. Birkin; Y. E. Watson; T. G. Leighton, "Efficient mass transfer from an acoustically oscillated gas bubble", Journal of the Chemical Society Chemical Communications. 2650-2651, (2001).

${ }^{21}$ M. Kornfeld; L. Suvorov, "On the destructive action of cavitation", Journal of Applied Physics. 15, 495-506, (1944).

${ }^{22}$ T. G. Leighton; D. G. Ramble; A. D. Phelps, "The detection of tethered and rising bubbles using multiple acoustic techniques", Journal of the Acoustical Society of America. 101, 2626-2635, (1997).

${ }^{23}$ A. D. Phelps; T. G. Leighton, "The subharmonic oscillations and combination-frequency subharmonic emissions from a resonant bubble: Their properties and generation mechanisms", Acustica. 83, 59-66, (1997).

${ }^{24} \mathrm{P}$. Tho; R. Manasseh; A. Ooi, "Cavitation microstreaming patterns in single and multiple bubble systems", Journal of Fluid Mechanics. 576, 191-233, (2007).

${ }^{25}$ W. E. Rowe; W.L.Nyborg, "Changes in the Electrode process brought about by small scale acoustic streaming", Journal of the Acoustics Society of America. 39, 965-971, (1966).

${ }^{26}$ W. L. Nyborg; M.I.L.Seegall. "Effects of acoustic microstreaming at electrodes"; Proceedings of the 3rd International Congress on Acoustics 1960, 346-348.

${ }^{27}$ A. I. Eller; L. A. Crum, "Instability of motion of a pulsating bubble in a sound field", Journal of the Acoustical Society of America. 47, 762-767, (1970).

${ }^{28}$ T. B. Benjamin; A. T. Ellis, "Self-propulsion of asymmetrically vibrating bubbles", Journal of Fluid Mechanics. 212, 65-80, (1990).

${ }^{29}$ P. Hall; G. Seminara, "Non-linear oscillations of non-spherical cavitation bubbles in acoustic fields", Journal of Fluid Mechanics. 101, 423-444, (1980).

${ }^{30}$ P. R. Birkin; Y. E. Watson; T. G. Leighton; K. L. Smith, "Electrochemical Detection of Faraday Waves on the Surface of a Gas Bubble.", Langmuir. 18, 2135-2140, (2002). 


\section{Birkin et al.}

${ }^{31}$ T. G. Leighton; R. J. Lingard; A. J. Walton; J. E. Field, "Acoustic bubble sizing by combination of subharmonic emissions with imaging frequency", Ultrasonics. 29, 319-323, (1991).

${ }^{32}$ T. G. Leighton "The Acoustic Bubble, pp 204-207, 306"; (Academic Press, London, 1994); 204-207, 306.

${ }^{33}$ M. A. Ainslie; T. G. Leighton, "Near resonant bubble acoustic cross-section corrections, including examples from oceanography, volcanology, and biomedical ultrasound", Journal of the Acoustical Society of America. 126, 2163-2175, (2009).

${ }^{34}$ M. A. Ainslie; T. G. Leighton, "Review of theories for scattering and extinction cross-sections, damping factors and resonance frequencies of spherical gas bubbles", Journal of the Acoustical Society of America. In press, (2011).

${ }^{35}$ T. G. Leighton "The Acoustic Bubble"; (Academic Press, London, 1994); 295.

${ }^{36}$ Y. E. Watson. "Electrochemical investigation of Bubble wall motion during non-inertial cavitation.". PhD, University of Southampton, 2003.

${ }^{37}$ A. Francescutto; R. Nabergoj, "Pulsation amplitude threshold for surface-waves on oscillating bubbles", Acustica. 41, 215-220, (1978).

${ }^{38}$ M. S. Plesset, "On the stability of fluid flows with spherical symmetry", Journal of Applied Physics. 25, 96-98, (1954).

${ }^{39}$ H. Lamb "Hydrodynamics"; (Cambridge University Press, Cambridge, 1932); 445-450.

${ }^{40}$ M. Versluis; D. E. Goertz; P. Palanchon; I. L. Heitman; S. M. van der Meer; B. Dollet; N. de Jong; D. Lohse, "Microbubble shape oscillations excited through ultrasonic parametric driving", Physical Review E. 82, 026321, (2010).

${ }^{41}$ R. G. Holt; D. F. Gaitan, "Observation of stability boundaries in the parameter space of single bubble sonoluminescence", Physical Review Letters. 77, 3791-3794, (1996).

${ }^{42}$ D. Zardi; G. Seminara, "Chaotic mode competition in the shape oscillations of pulsating bubbles", Journal of Fluid Mechanics. 286, 257, (1995).

${ }^{43}$ R. Mettin. From a single bubble to bubble structires in acoustic cavitation. In Oscillations, Waves and Interactions; Kurz, T., Parlitz, U., Kaatze,

U., Eds.; Universitätsverlag Göttingen: Göttingen, 2007; pp 171-198.

${ }^{44}$ N. Bremond; M. Arora; C. D. Ohl; D. Lohse, "Cavitating bubbles on patterned surfaces", Physics of Fluids. 17, 091111, (2005).

${ }^{45}$ A. O. Maksimov; T. G. Leighton; P. R. Birkin, "Dynamics of a tethered bubble", Innovations in Nonlinear Acoustics. 838, 512-515, (2006).

${ }^{46}$ D. G. Ramble; A. D. Phelps; T. G. Leighton, "On the relation between surface waves on a bubble and the subharmonic combination-frequency emission", Acustica. 84, 986-988, (1998).

${ }^{47}$ A. O. Maksimov; T. G. Leighton, "Transient processes near the threshold of acoustically driven bubble shape oscillations", Acta Acustica. 87, 322-332, (2001).

${ }^{48}$ J. Kwak; A. J. Bard, "Scanning electrochemical microscopy - theory of the feedback mode", Analytical Chemistry. 61, 1221-1227, (1989).

${ }^{49}$ D. G. Offin. "An investigation of fast surface re-formation in the presence of inertial (transient) cavitation". University of Southampton, 2006. 


\section{Birkin et al.}

${ }^{50}$ T. G. Leighton; P. R. Birkin; M. Hodnett; B. Zeqiri; J. F. power; G. J. Price; T. Mason; M. Plattes; N. Dezhkunov; A. Coleman. Cavitation of measurements of reference acoustic cavitation (COMORAC): An experimental feasibility trial. In Bubble and Particle Dynamics in Acoustic Fields: Modern Trends and Applications; Doinikov, A. A., Ed.; Research Signpost: Kerala, 2005; pp 37-94.

${ }^{51}$ T. J. Mason; J. P. Lorimer; D. M. Bates, "Quantifying Sonochemistry - casting some light on a black art", Ultrasonics. 30, 40-42, (1992).

${ }^{52}$ E. Maisonhaute; B. A. Brookes; R. G. Compton, "Surface acoustic cavitation understood via nanosecond electrochemistry. 2 . The motion of acoustic bubbles", Journal of Physical Chemistry B. 106, 3166-3172, (2002).

${ }^{53}$ E. Maisonhaute; P. C. White; R. G. Compton, "Surface acoustic cavitation understood by nanosecond electrochemistry", Journal of Physical Chemistry B. 105, 12087-12091, (2001).

${ }^{54}$ P. R. Birkin; D. G. Offin; P. F. Joseph; T. G. Leighton, "Cavitation, shock waves and the invasive nature of sonoelectrochemistry", Journal of Physical Chemistry B. 109, 16997-17005, (2005).

${ }^{55}$ T. J. Asaki; P. L. Marston, "Free decay of shape oscillations of bubbles acoustically trapped in water and sea water", Journal of Fluid Mechanics. 300, 149-167, (1995).

${ }^{56}$ Y. E. Watson; P. R. Birkin; T. G. Leighton, "Electrochemical detection of Bubble Oscillation", Ultrasonics Sonochemistry. 10, 65-69, (2003).

${ }^{57}$ A. O. Maksimov; T. G. Leighton, "Pattern formation on the surface of a bubble driven by an acoustic field", Proceedings of the Royal Society A. In press doi:10.1098/rspa.2011.0366, (2011).

${ }^{58}$ A. O. Maksimov, "On the volume oscillations of a tethered bubble", Journal of Sound and Vibration. 283, 915-926, (2005).

${ }^{59}$ See supplementary material at [URL will be inserted by AIP] for ring-down of the bubble on the surface at different stages during this process.

${ }^{60}$ K. Yoshida; Y. Watanabe, "Variation of periodicity in non-spherical bubble vibration", Innovations in Nonlinear Acoustics. 479, 512, (2006).

${ }^{61} \mathrm{CRC}$ "Handbook of Chemistry and Physics 1913-1995", 75th ed.; (CRC Press, 1995); 6-1, 6-20.

${ }^{62}$ G. W. C. Kaye; T. H. Laby "Tables of Physical and Chemical Constants and some Mathematical Functions", 12th ed.; (Longmans, Green and Co, London, 1959); 1-231. 


\section{Figure Legends}

Figure 1. Collection of results showing the bubble oscillation recorded with either imaging $(a, b)$ or electrochemical technology (c). Frame (a) shows a side on image of an air bubble trapped on a copper plate (scale bar $=2 \mathrm{~mm}$ ), frame (b) an image from below a different air bubble trapped on a glass rod (bubble $\sim 4 \mathrm{~mm}$ in diameter). In both (a) and (b) Faraday waves can be seen on the gas/liquid interface. Plot (c) shows how the average current $\left(i_{\mathrm{av}}, \bullet\right)$ for a $25 \mu \mathrm{m}$ diamter microelectrode varied as a function of distance away from the a bubble (different to (a) and (b)) driven into surface wave motion (note the error bar represents the $95 \%$ confidence interval). The dotted horizontal line in the main plot represents the current recorded in the bulk liquid in the absence (e.g. in the absence of an impossed sound field) of forced convection (microstreaming). Note the inset shows the actual current (i) time history (-) recorded in one position compared to the zero-to-peak acoustic pressure signal (- - ). The solution temperature was $20-25{ }^{\circ} \mathrm{C}$.

Figure 2. Schematic representation (not to scale) of a bubble moving over the surface of an electrode. The figure shows the position of the sound source (horn tip, $\mathrm{H}$ ) with respect to the electrode. In this example the electrode is maintained at a constant separation, d, (e.g. $4.5 \mathrm{~mm})$ from the sound source. The source operartes as a piston like emitter with an oscillation amplitude $A_{d}$. The microelectrode is held under potential control with respect to a reference electrode (not shown). The potential chosen oxidises compount $A$ to $B$ and the current that passes $(i)$ is recorded as a function of time (for example). Forced convection (e.g. microstreaming) caused by a bubble trapped on the surface (shown to be driven into surface wave oscillation) causes an enhancement (and periodicity in) the current recorded. The solution also contains an inert electrolyte. Lastly a counter electrode (not shown) passes the opposite electrochemical current to the microelectrode at all times. 
Figure 3. (a) Plot showing the current time traces recorded simultaneously from the $125 \mu \mathrm{m}$ diameter lead $\left.\left(i_{\mathrm{Pb}}\right)\right)$ and $50 \mu \mathrm{m}$ diameter platinum $\left(i_{\mathrm{Pt}}\right)$ microdiscs of a dual electrode under exposure to ultrasound $\left(22.83 \mathrm{kHz}\right.$, nominal faceplate intensity $56 \pm 5 \mathrm{~W} \mathrm{~cm}^{-2}, \sim 30 \mathrm{kPa}$ zero-topeak pressure amplitude at $4.5 \mathrm{~mm}$ below the horn). The electrode-to-horn separation was 4.5 mm. The solution contained $20 \mathrm{mmol} \mathrm{dm}{ }^{-3} \mathrm{~K}_{4} \mathrm{Fe}(\mathrm{CN})_{6}$ and $0.75 \mathrm{~mol} \mathrm{dm}^{-3} \mathrm{Na}_{2} \mathrm{SO}_{4}$ and the experiment was performed under aerobic conditions. The potentials of both discs was held at +0.8 V vs. Ag. The initial solution temperature was $20-25{ }^{\circ} \mathrm{C}$. (b) Frames from a high-speed camera imaging the space above the dual electrode employed in (a). The capture rate was 60000 fps. The images (not temporally correlated to (a)) were taken from a 'side-on' perspective with respect to the electrode/solution interface. The microelectrode wires can be seen at the bottom of each frame. The platinum (50 $\mu \mathrm{m}$ diameter) mass transfer sensor is on the left and the lead (125 $\mu \mathrm{m}$ diameter) erosion sensor is on the right. The white scale bar in frame 1 represents $175 \mu \mathrm{m}$. A bubble trapped on the surface of the electrode is labelled with 'B' on frame 1.

Figure 4(a). Acoustic Pressure $(P)$ time profile for a hydrophone placed in the cell $(\sim 3 \mathrm{~cm}$ from tip of ultrasonic source). $\mathbf{4 ( b )}$ Current (i) recorded at a $25 \mu \mathrm{m}$ diameter platinum electrode in a solution of $2 \mathrm{mM} \mathrm{KI}$ and $0.1 \mathrm{M} \mathrm{KCl}$ during a ring-down experiment (see main text for full details). The potential of the electrode was held at $+0.6 \mathrm{~V} v s$. SCE. The dotted line shows the time at which the ultrasound $\left(22.83 \mathrm{kHz}\right.$, nominal faceplate intensity $56 \pm 5 \mathrm{~W} \mathrm{~cm}^{-2}, \sim 30 \mathrm{kPa}$ zero-to-peak pressure amplitude at $4.5 \mathrm{~mm}$ below the horn) was terminated. The temperature measured after the experiment was complete was $40 \pm 3{ }^{\circ} \mathrm{C}$ (elevated temperature due to intense lighting necessary for high-speed imaging). The frame numbers given on the top axis relate to Figure 5. The electrode to horn separation was $4.5 \mathrm{~mm}$. 
Figure 5. Images of 200 frames showing the bubble behaviour during a ring-down experiment. The images were taken from a 'side-on' perspective with respect to the electrode/solution interface. The inter-frame time is $0.5 \mathrm{~ms}$ (every $50^{\text {th }}$ frame from data recorded at $100000 \mathrm{fps}$ ). These images were recorded simultaneously with the data shown in Fig. 4. The frames read left to right top to bottom. The frame number is indicated by the sum of appropriate row and column labels. The first frame is at $t=0.007 \mathrm{~ms}$. The drive to the transducer $(22.83 \mathrm{kHz}$, nominal faceplate intensity $56 \pm 5 \mathrm{~W} \mathrm{~cm}^{-2}, \sim 30 \mathrm{kPa}$ zero-to-peak pressure amplitude at $4.5 \mathrm{~mm}$ below the horn) was terminated after $\sim 15 \mathrm{~ms}$ (frame 30 ). See supplementary material on the Journal of the Acoustical Society of America (JASA) website ${ }^{59}$ for examples of movie sequences of bubble ringdown.

Figure 6 a-d Image sequences showing the bubble behaviour at increasing times during a ringdown experiment. The images were taken from a 'side-on' perspective with respect to the electrode/solution interface. In all cases the capture rate was $100000 \mathrm{fps}$. The time for the first frame $\left(t_{1 \mathrm{st}}\right)$ in each sequence is as follows (where $t=0 \mu \mathrm{s}$ is the trigger point): (a) $t_{1 \mathrm{st}}=9287 \mu \mathrm{s}$, (b) $t_{1 \mathrm{st}}=27187 \mu \mathrm{s}$, (c) $t_{1 \mathrm{st}}=67007 \mu \mathrm{s}$, (d) $t_{1 \mathrm{st}}=81717 \mu \mathrm{s}$. The $25 \mu \mathrm{m}$ diameter Pt wire is shown in each frame (bottom left). Each frame is $\sim 350 \mu \mathrm{m}$ across. The drive to the transducer $(22.85 \mathrm{kHz}$, $56 \pm 5 \mathrm{~W} \mathrm{~cm}^{-2}, \sim 30 \mathrm{kPa}$ zero-to-peak pressure amplitude at $4.5 \mathrm{~mm}$ below the horn) was terminated at $t=15 \mathrm{~ms}$. In each sub-figure the frames read left to right top to bottom. The frame number is indicated by the sum of appropriate row and column labels. See supplementary material on the Journal of the Acoustical Society of America (JASA) website ${ }^{59}$ for examples of movie sequences of bubble ring-down. 
Figure 7. (a) An expanded view of Fig. 6(d) frame 79. The white dashed line shows the outline of an extreme motion of the $l=5$ zonal spherical harmonic perturbation with a vertical axis of symmetry. (b) The shape of the octahedral (cube) mode for $l=4$. The left and right figures correspond to maximal distortions induced by the distortion modes. In dimensionless units, the equilibrium bubble radius is $R_{0}=1$, the amplitudes of $\left(Y_{l, m}, l=4, m=4\right) a_{4,4}$ and $(l=4, m=0)$ $a_{4,0}$ modes $=1.67 a_{4,4}=0.06 \quad\left(Y_{l, m}\right.$ is a spherical harmonic). The best fit was realized for $a_{(4,0)} / R_{0}=0.06$. (c) Illustration of the influence of the rigid wall on the shape of the $l=4$ bubble oscillation mode shown in (b), when the bubble is close to (the left panel) and touching (the right panel) the wall.

Figure 8 Plot showing the predicted acoustic zero-to peak pressure amplitude thresholds $\left(P_{l}\right.$ threshold) for the onset of surface waves for modes $l=2(-), 3(\cdots \cdot), 4(---), 5(-\cdot \cdot \cdot \cdot), 6(--$ - ) and $7(-\cdot-\cdot-)$ (calculated using the parameters in Table 1). 
Parameter

Equilibrium radius $\left(R_{0}\right)$

Static pressure at the bubble $\left(P_{0}\right)$
Density $(\rho)$

Viscosity $(\eta)$

Surface tension $(\sigma)$

Speed of sound $(c)$

Vapour pressure $\left(P_{\mathrm{v}}\right)$

\section{Value}

$110 \pm 10 \mu \mathrm{m}$

$1.01 \times 10^{5} \mathrm{~Pa}$

Liquid properties

\section{Gas Properties}

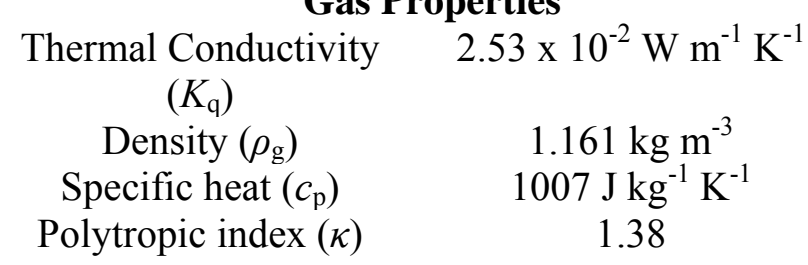

Table 1 Physical parameters ${ }^{61,62}$ used in construction of figure 8 . 


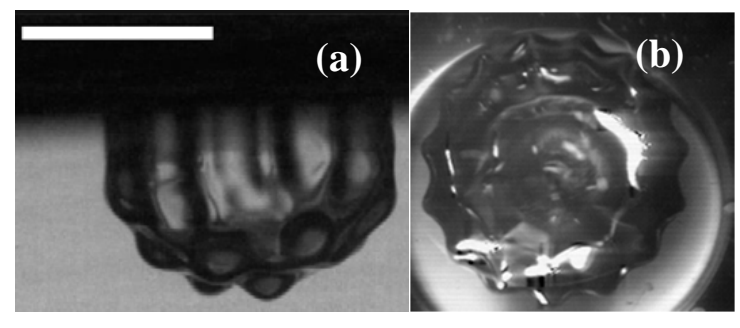

(c)

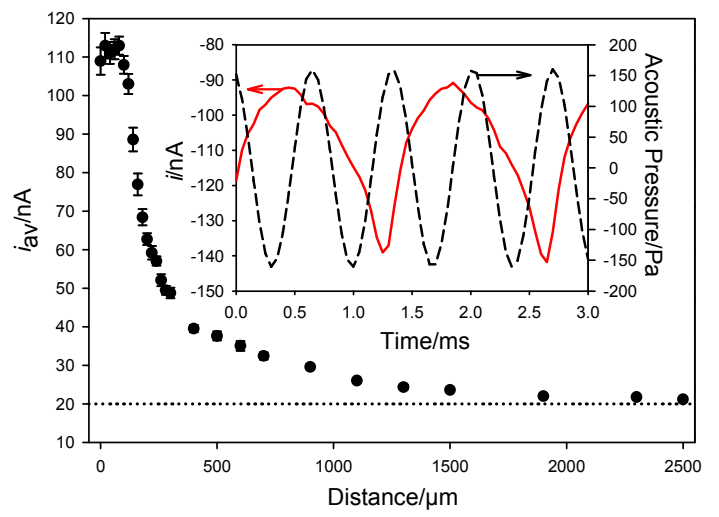

Figure 1. 
Birkin et al.

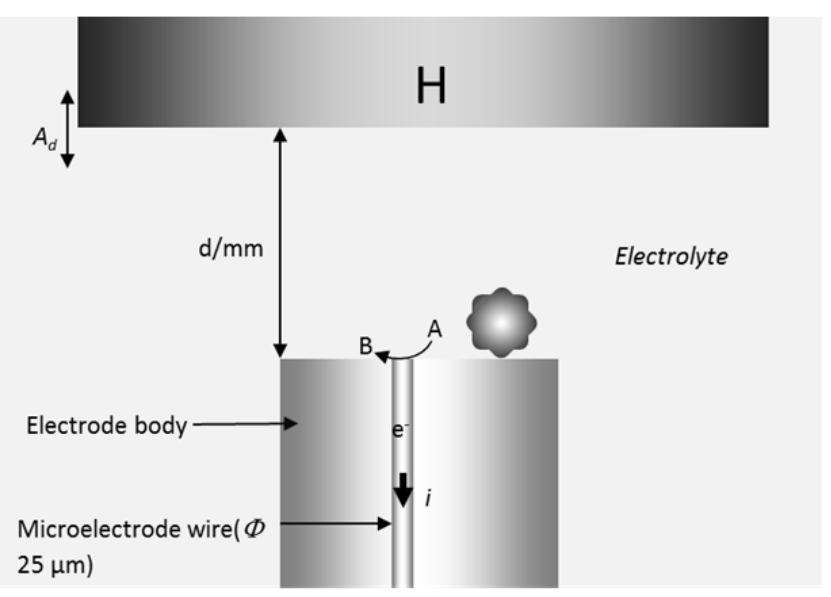

Figure 2. 
Birkin et al.

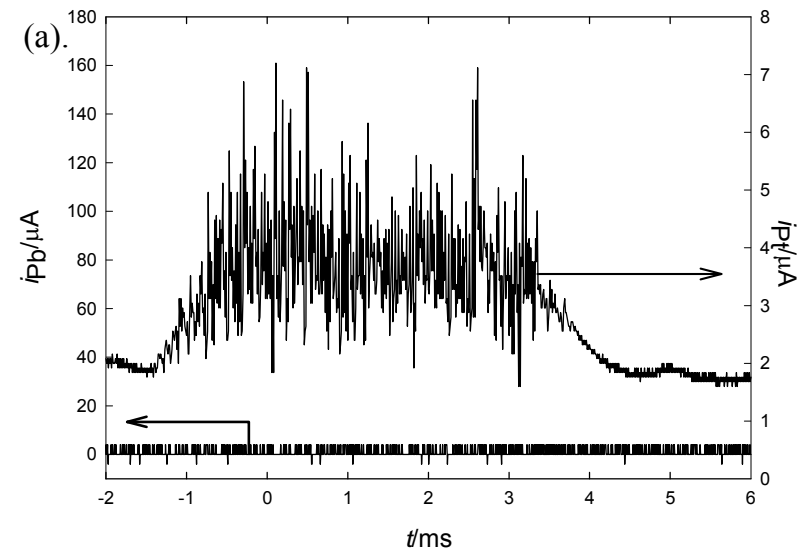

(b).

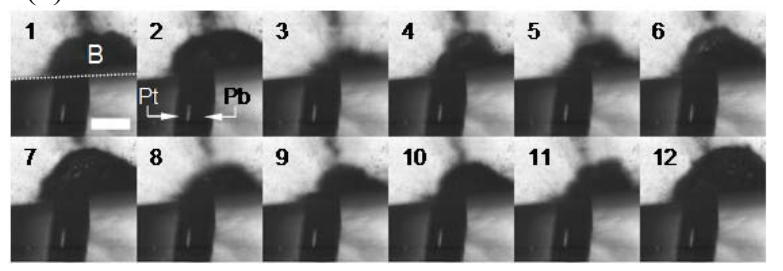

Figure 3 
Birkin et al.
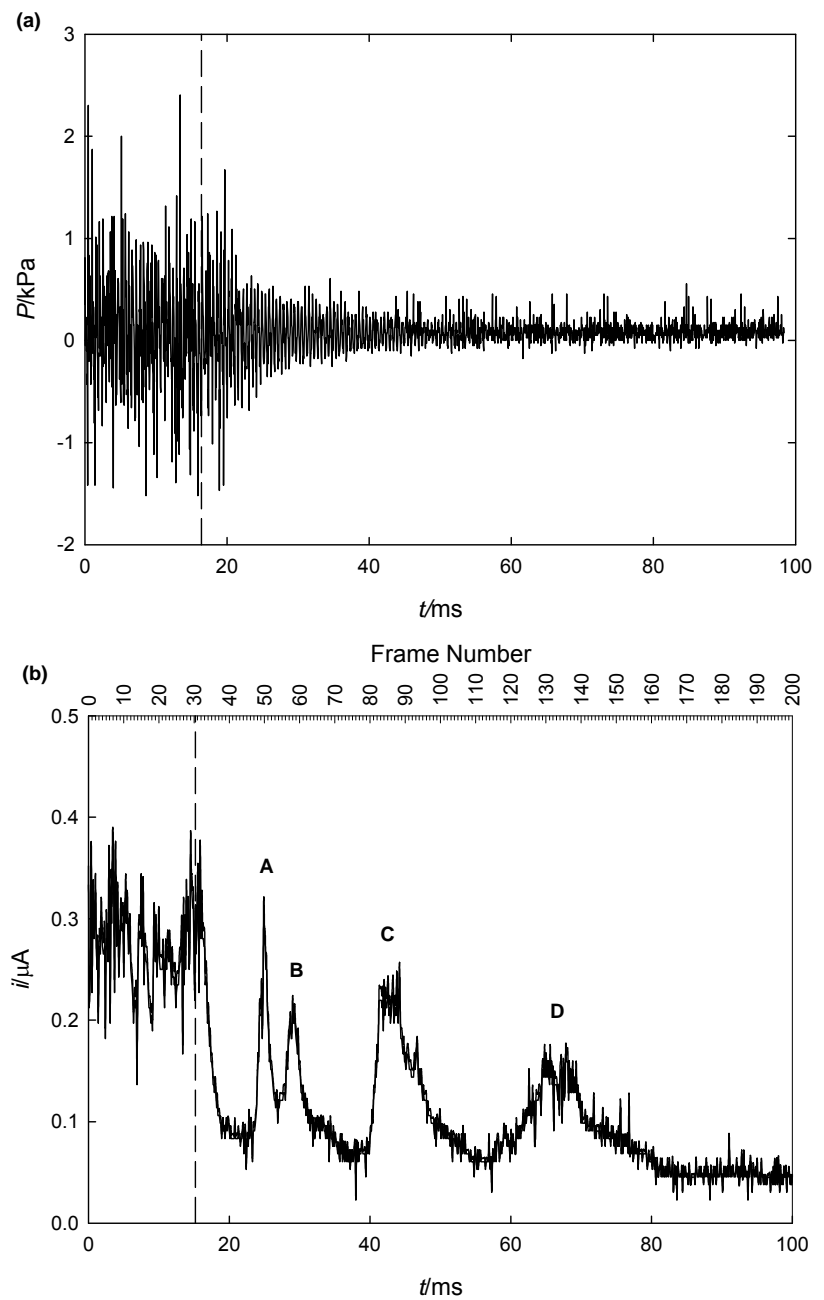

Figure 4 
Birkin et al.

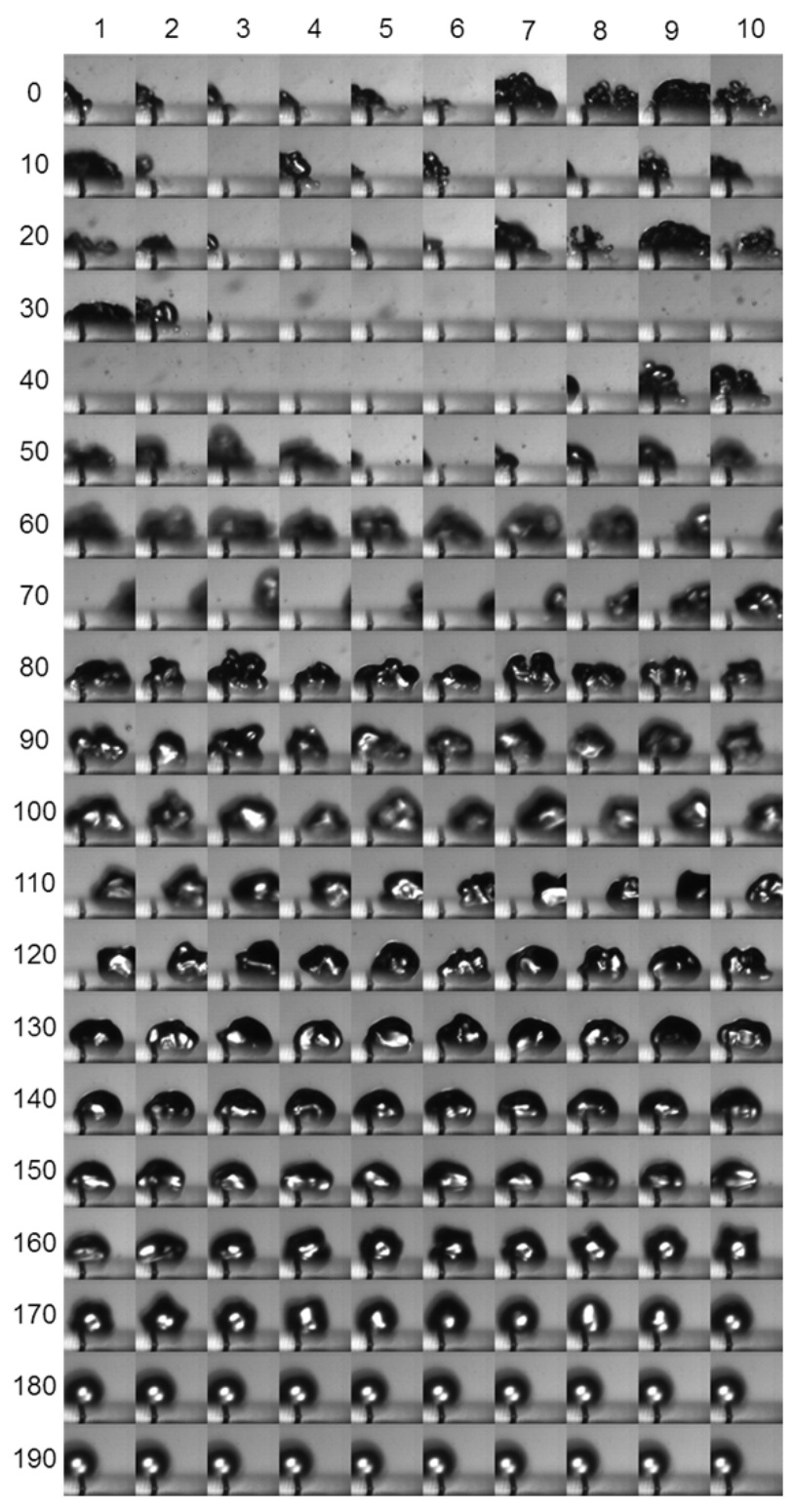

Figure 5 


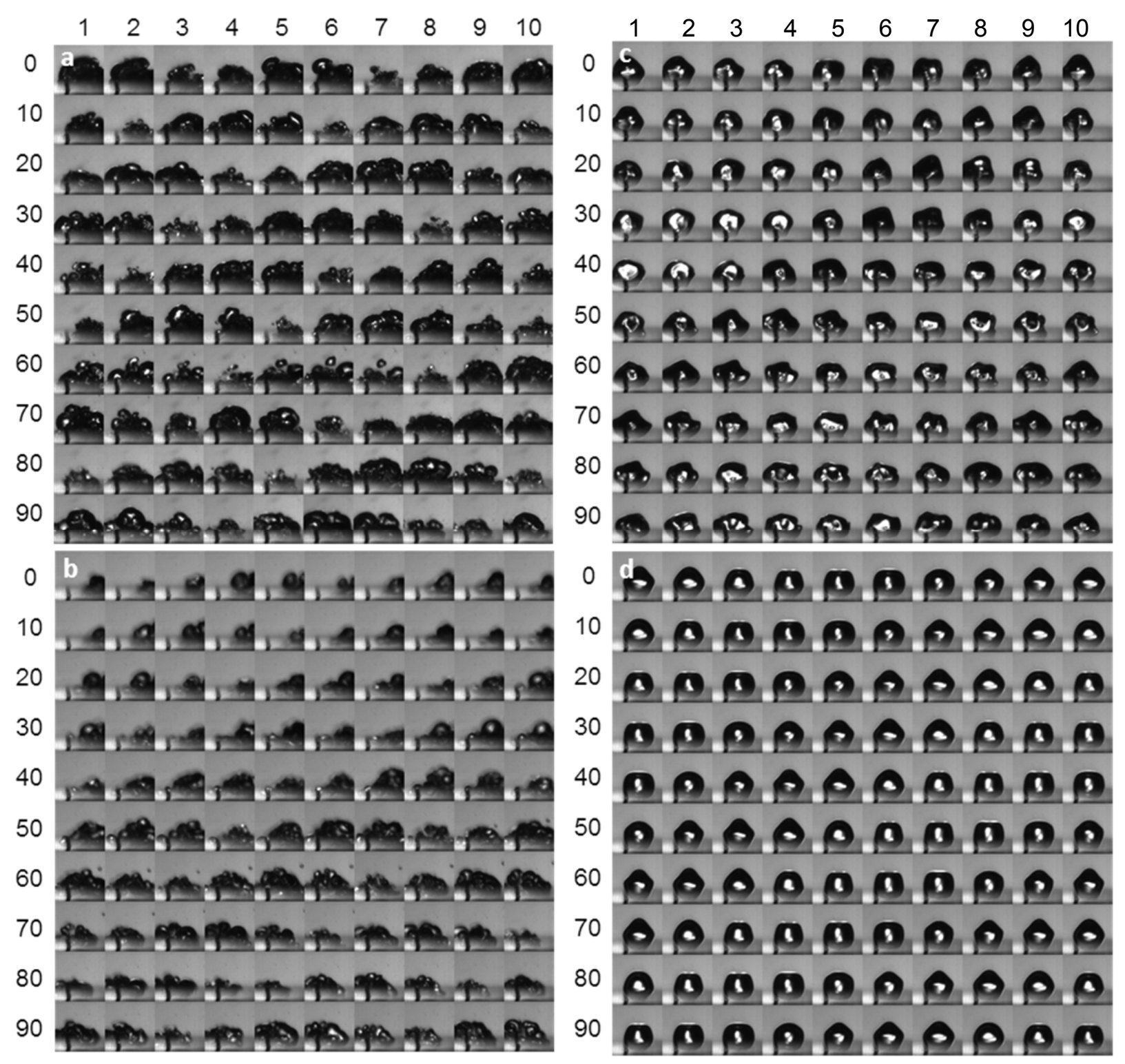

Figure 6 
(a)

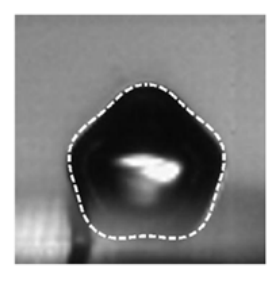

(b)
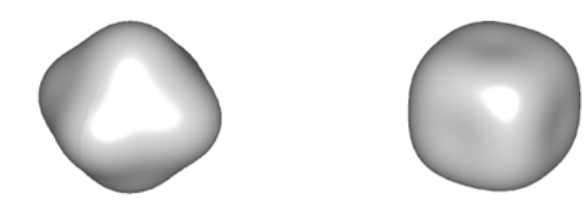

(c)
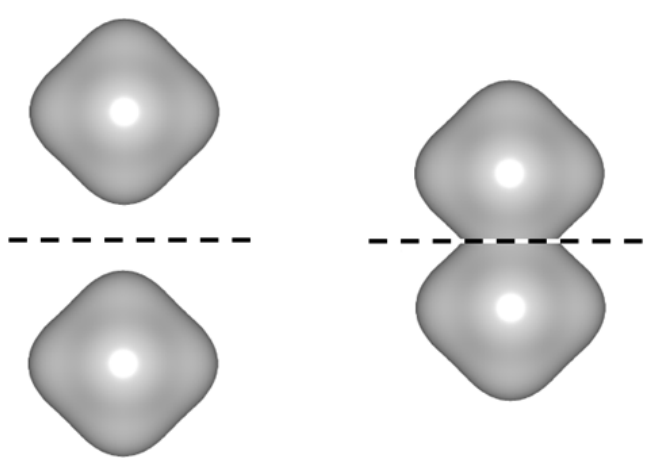

Figure 7 


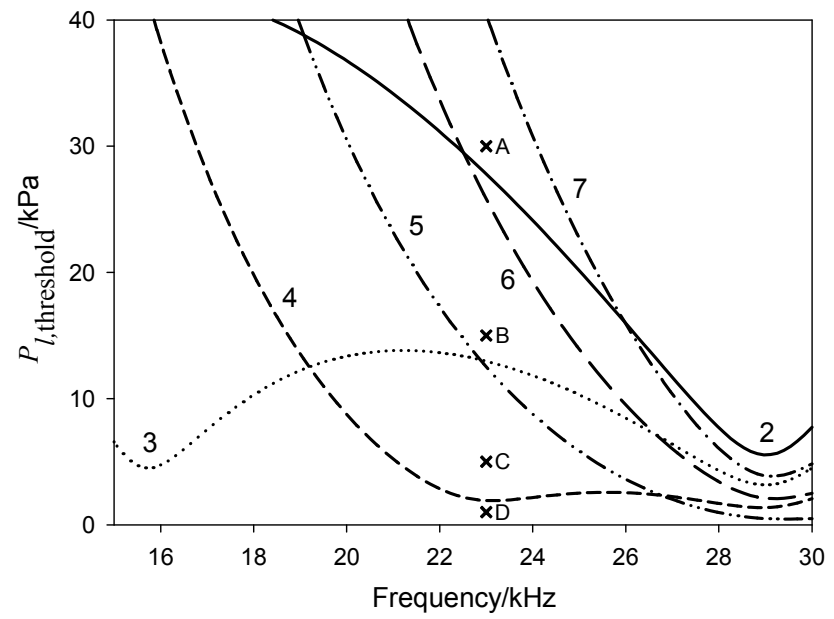

Figure 8 